\title{
Propuesta metodológica para la evaluación de competencias transversales en el Máster en Ingeniería Mecánica de la Universitat Politècnica de València
}

\author{
J. Carballeiraa ${ }^{\text {a1 }}$, M.Tur ${ }^{1}$, A.J. Besa ${ }^{1}$, J. Albelda ${ }^{1}$, J.E. Tarancón ${ }^{1}$, J. Martínez-Casas ${ }^{1}$, F.D. Denia ${ }^{1}$, \\ J.J. Ródenas ${ }^{1}$
}

a(jacarmo@mcm.upv.es)

${ }^{1}$ Departamento de Ingeniería Mecánica y de Materiales, Universitat Politècnica de València (UPV), Camino de Vera $\mathrm{s} / \mathrm{n}^{\mathrm{o}}, 46022$ Valencia.

\begin{abstract}
This contribution presents the work carried out within the framework of an educational innovation and improvement project developed during the last two years in the Master's Degree in Mechanical Engineering at the Technical University of Valencia. One of the main objectives of this project is the development and implementation of new methodologies for the evaluation of generic competences. Among these new methodologies, there is an approach through projectbased learning, which allows for the incorporation of the assessment of some generic competences that was not done previously in a proper way. Therefore, several subjects have been coordinated, a new type of Master's Thesis has been proposed, with the collaboration of a company, and new assessment tools have been designed.
\end{abstract}

Keywords: assessment, generic competences, Master's Thesis

\section{Resumen}

En esta comunicación se presentan los trabajos desarrollados en el marco de un proyecto de innovación y mejora educativa llevado a cabo durante los dos últimos cursos en el Máster Universitario en Ingeniería Mecánica de la Universitat Politècnica de València. Uno de los principales objetivos de este proyecto es el desarrollo y puesta en marcha de nuevas metodologías para la evaluación de competencias transversales. Entre estas nuevas metodologías está una aproximación mediante el aprendizaje basado en proyectos, que permite incorporar la evaluación de algunas competencias transversales que no se hacia de forma adecuada con anterioridad. En esta linea se han coordinado varias asignaturas, se ha planteado un nuevo tipo de Trabajo Fin de Máster, con la colaboración de una empresa, y se han diseñado nuevas herramientas de evaluación.

Palabras clave: evaluación, competencias transversales, trabajo fin de máster. 


\section{Introducción}

El Máster Universitario en Ingeniería Mecánica se imparte en la Universitat Politècnica de València (UPV) con el actual plan de estudios desde el curso 2014-2015 (y anteriormente con otro plan de estudios desde el curso 2009-2010). Tal y como se recoge en los informes de gestión del título de los últimos años, el máster presenta, en general, unos excelentes resultados en cuanto a satisfacción del alumnado y titulados con la labor del profesorado y la gestión general del máster. Asimismo presenta también buenos indicadores en cuanto a tasa de eficiencia, tasa de rendimiento e internacionalización. Existen, sin embargo, algunos aspectos que son susceptibles de mejora.

Por un lado, la tasa de graduación es menor de la prevista en la memoria de verificación del título. Un análisis llevado a cabo por la Comisión Académica del Título (CAT) mostró que un porcentaje de estudiantes no finalizaba el Trabajo Fin de Máster (TFM) en el plazo previsto. Al parecer, algunos de los TFM ofertados tienen un carácter investigador que no coincide con los intereses de algunos estudiantes en ciertas ocasiones. Por consiguiente, cuando empiezan unas prácticas en empresa, descuidan el Trabajo y su realización se dilata en el tiempo.

Por otro lado, desde la implantación del máster se han ido incorporando en las diferentes asignaturas la evaluación de competencias transversales. Sin embargo existen unas competencias transversales que no se evaluaban de forma adecuada: CT06 Trabajo en equipo y liderazgo; CT07 Responsabilidad ética medioambiental y profesional; CT10 Conocimiento de problemas contemporáneos; CT11 Aprendizaje permanente; y CT12 Planificación y gestión del tiempo, empleando las denominaciones del proyecto institucional de la UPV en competencias transversales (UPV, 2014).

Con estos aspectos en mente se planificó el proyecto de innovación y mejora educativa (PIME) cuyos resultados preliminares se presentan en esta contribución. A partir de los trabajos desarrollados en este ámbito por una parte de los autores, en calidad de componentes de un Equipo de Innovación y Calidad Educativa de la UPV (EICE), se tenía claro que había que introducir metodologías activas, tanto en las asignaturas, como en la realización del propio TFM (Carballeira, 2017). La idea principal fue definir un trabajo fin de máster que permitiera resolver ambas situaciones: aumentando la motivación de los estudiantes, e incorporando las competencias transversales mencionadas en su desarrollo y evaluación. Y para preparar a los estudiantes en este modo de aprendizaje, se coordinarían las asignaturas del Máster para realizar al menos un proyecto entre varias de ellas.

\section{Objetivos}

Los objetivos principales de esta innovación son:

- La definición de un TFM motivador y aplicado, que permita a los estudiantes tener la certeza de que se va a llevar a cabo en un plazo razonable, y a la vez suponga una oportunidad para seguir desarrollando y evaluando sus competencias transversales.

- La coordinación de asignaturas del Máster para proponer al menos un proyecto multidisciplinar dentro del mismo.

- La planificación de una metodología y el desarrollo de nuevas herramientas para la evaluación de las competencias transversales mencionadas en la Introducción. 


\section{Desarrollo de la innovación}

Para el TFM se propone resolver un 'reto de diseño', en sintonía con la temática del máster que tiene su núcleo principal en la simulación y el diseño mecánico de sistemas. Se planificará su desarrollo mediante una aproximación al aprendizaje basado en proyectos. Se establecerá una competición en la que se planteará un problema concreto de diseño con unas normas, restricciones y requisitos establecidos y se formarán grupos de estudiantes que propondrán soluciones alternativas al reto. El sistema se dividirá en tres o cuatro componentes que puedan dan lugar a trabajos fin de máster individuales, pero relacionados entre sí.

Esta metodología es adecuada para desarrollar las competencias transversales propuestas mediante un aprendizaje cooperativo (Villa, 2011). El reto planteado, relacionado con un problema real y con múltiples soluciones posibles, debe servir de motivación adicional para que el estudiante busque y aprenda los conocimientos necesarios para resolver con éxito el diseño (Mettas, 2007).

La CT06, la CT07 y la CT12 están claramente involucradas en la realización de un proyecto coordinado entre varios estudiantes y con un nivel de complejidad elevado. Para trabajar las competencias CT10 y CT11 se planteará un 'reto de diseño' sobre un problema de actualidad y que requiera aprendizajes novedosos. Para la evaluación de estas competencias se adaptarán las rúbricas publicadas por la UPV en su proyecto institucional (UPV, 2014), y se estudiarán otras experiencias en este terreno (Ye-Lin, 2017).

Existen en el máster algunas asignaturas cuyo contenido se puede estudiar en base a proyectos del estilo de 'reto de diseño'. En estas asignaturas se utilizan herramientas de simulación y análisis para realizar diseños mecánicos bajo diferentes condiciones:

- Diseño asistido por ordenador mediante el método de los elementos finitos: Simulación de sólidos deformables con comportamiento elástico lineal.

- Diseño con materiales compuestos: Simulación de materiales compuestos y cálculo de la integridad estructural.

- Fatiga y tolerancia al fallo. Integridad estructural en condiciones de carga variable.

- Mecánica estructural no lineal: Simulación de componentes en contacto y, con grandes desplazamientos.

- Diagnóstico y corrección de fallos de componentes mecánicos y Acústica y control de ruido: Análisis mediante vibraciones del comportamiento de sistemas mecánicos.

- Dinámica de vehículos: Simulación de vehículos automóviles y vehículos ferroviarios.

Anteriormente, una parte de la evaluación de estas asignaturas se basa en un trabajo tradicional realizado por los estudiantes. De este modo, se coordinarán algunas de las asignaturas para que propongan un proyecto común, cuya resolución implique un aprendizaje basado en el proyecto. Los estudiantes utilizarán el proyecto planteado como punto de partida para adquirir e integrar nuevos conocimientos relacionados con la materia que se tiene que estudiar en cada asignatura (Barrows, 1986). 


\section{Resultados}

Para dar respuesta a los objetivos de esta innovación, se han obtenido los siguientes resultados preliminares. En primer lugar, se ha ofertado un TFM en colaboración con la empresa STADLER, que es un fabricante de vehículos ferroviarios con una factoría cercana a la universidad. En este Trabajo los estudiantes se enfrentan a un desafío real para el diseño de una estructura cuyas restricciones y especificaciones serán facilitadas por la empresa. Tienen que desarrollar el Trabajo en equipo y su desempeño es evaluado por un tribunal con miembros que pertenecen a la empresa, y que otorgará un premio económico a las mejores propuestas. La convocatoria se encuentra publicada aquí (Cátedra Stadler, 2020).

Para el presente curso, el reto consiste en el diseño mecánico de la estructura de una locomotora de maniobras. Este diseño supone el cumplimiento de una serie de normativas sobre seguridad e integridad estructural, además de un conjunto de requisitos de la empresa acerca de la geometría, materiales, etc. En la figura 1 se puede ver una imagen de este tipo de locomotoras. En la figura 2 se muestra uno de los resultados obtenidos en uno de los primeros trabajos fin de máster realizados.

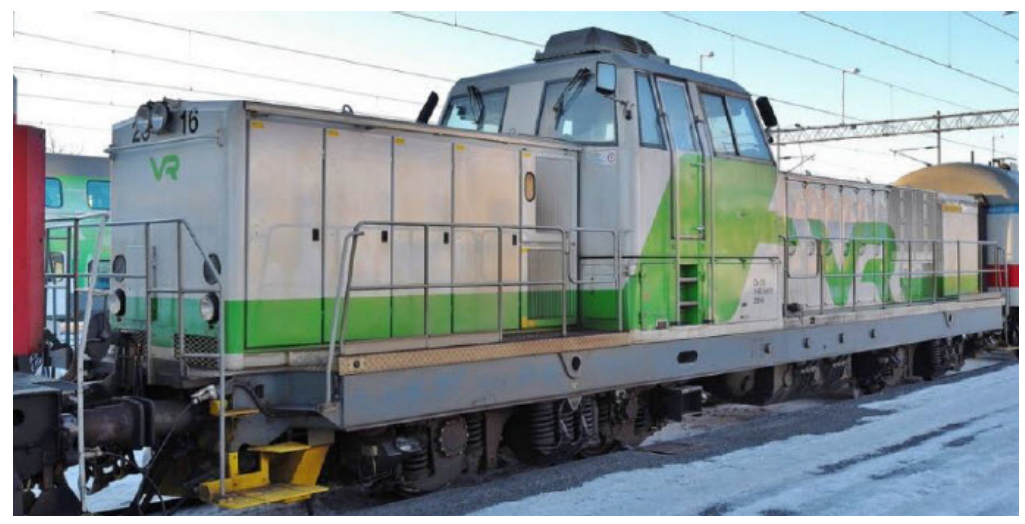

Fig. 1 Locomotora de maniobras VR LOCOMTIVE modelo DR16
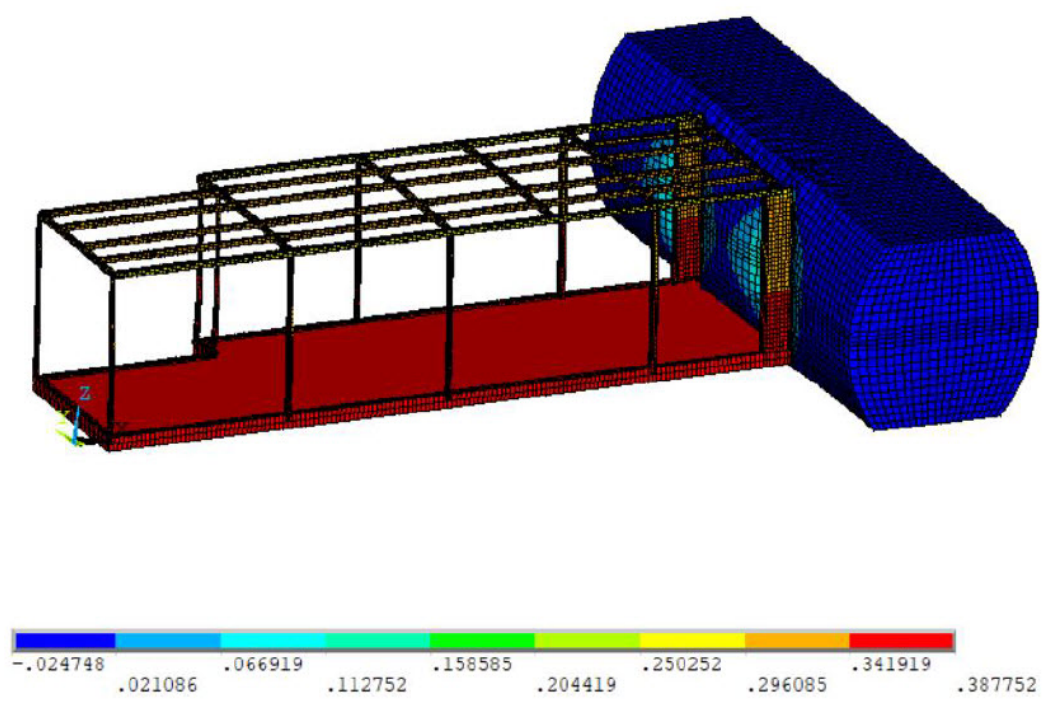

Fig. 2 Desplazamiento longitudinal de la estructura de la capota larga en una simulación de un impacto contra un camión cisterna 
En segundo lugar, se han coordinado las asignaturas de "Fatiga y tolerancia al fallo" y de "Mecánica Estructural No Lineal" para llevar a cabo un proyecto en común que suponga más del $50 \%$ de la nota final de la asignatura. Se trata de un trabajo que se llevará a cabo en equipos y con una metodología similar a la que se plantea para el TFM "Reto de diseño".

Finalmente, para evaluar las competencias transversales en el TFM, se ha planificado una estrategia y se han preparado instrumentos partiendo de las rúbricas desarrolladas por los equipos de trabajo del Instituto de Ciencias de la Educación de la UPV (ICE).

Será el tutor del TFM el encargado de cuantificar el grado de cumplimiento de los indicadores utilizados en cada competencia, utilizando diferentes metodologías:

1. Evaluación entre iguales. Para la coevaluación el tutor proporcionará un cuestionario a cada componente del equipo. Este cuestionario se completará y enviará al tutor al finalizar el proyecto.

2. Autoevaluación. En el mismo formulario habrá cuestiones relacionadas con el desempeño personal.

3. El tutor realizará la evaluación a partir de reuniones periódicas, cada 2-4 semanas y la información recibida por parte de los miembros del equipo.

En ciertas competencias el tribunal del TFM también hará la evaluación analizando la documentación presentada y realizando las cuestiones que estime oportunas en la exposición oral.

A modo de ejemplo, se presenta la estrategia y los instrumentos de evaluación para la competencia transversal CT07 (Responsabilidad ética, medioambiental y profesional).

A partir de los indicadores de la rúbrica de la UPV para el nivel de Máster:

- CT07-I1. Satisface, mediante el diálogo, alguna necesidad vinculada a la convivencia a partir de los valores éticos deseados.

- CT07-I2: Coordina acciones integrales respetuosas en el ámbito profesional.

- CT07-I3. Evalúa actuaciones integrales profesionales de acuerdo con los recursos disponibles materiales y humanos en términos de respeto con el entorno social, económico y ambiental.

Se plantean las siguientes herramientas y cuál es la estrategia para recopilar las evidencias. Para el primer indicador:

- Alumnos: Deben completar un cuestionario de autoevaluación y co-evaluación, que se entregará al tutor, quien será el responsable de valorar la competencia. En el cuestionario se preguntará si ha surgido algún conflicto en el equipo

- Tutor: En caso de que haya existido algún conflicto o diferencias de criterio, el tutor en una reunión con los miembros del equipo preguntará cómo han resuelto la situación.

Para el segundo indicador:

- Alumnos: Cuestionario sobre la actitud de los otros miembros del equipo respecto a: cumple con las tareas asignadas en tiempo y forma; participa activamente en las decisiones acerca de plazos y objetivos; acepta las normas y no busca atajos para conseguir finalizar el proyecto.

- Tutor: Identificar si cumplen con las tareas asignadas y siguen la normativa del trabajo: uso de software legal, etc.

Para el tercer indicador, se plantea la participación del Tribunal, que valorará a partir, tanto de la información del documento del TFM, como de las preguntas que formule durante la defensa si se ha tenido en cuenta, además de criterios económicos, criterios de sostenibilidad en la elección de materiales y procesos de fabricación, por ejemplo.

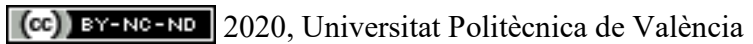

Congreso In-Red (2020) 


\section{Conclusiones}

En esta comunicación se presentan los resultados preliminares de un proyecto de innovación y mejora educativa que se ha llevado a cabo en el Máster Universitario en Ingeniería Mecánica de la UPV. Estos resultados incluyen la oferta de un nuevo TFM "Reto de diseño" en colaboración con la empresa Stadler, la realización este curso de un proyecto común entre dos asignaturas con una aproximación al aprendizaje basado en proyectos, y la generación de una estrategia y materiales para la evaluación de competencias transversales durante el TFM.

Las principales conclusiones que se pueden extraer del trabajo realizado hasta el momento son:

- Los estudiantes que han escogido el TFM ofertado se han mostrado muy satisfechos con el desafío planteado. Aunque el desarrollo se ha visto entorpecido por la situación creada por el COVID-19, los primeros trabajos presentados tienen una calidad elevada.

- Las herramientas generadas para la evaluación de las competencias transversales proporcionan una guía a los estudiantes acerca de los resultados de aprendizaje que se esperan de su trabajo. Esto supone una mejora frente a la situación anterior en la que no existían dichas referencias para orientar su esfuerzo y aprendizaje. En futuros trabajos se comparará la situación inicial de los estudiantes en cuanto al desarrollo de estas competencias transverales, con la situación final tras la realización del TFM, con objeto de mejorar las herramientas propuestas.

- La estrategia para la evaluación de las competencias transversales, obtenida como resultado de este proyecto de innovación, implica que todos los actores: tutor, estudiante y tribunal, comparten la misma definición de dichas competencias, lo que facilita mucho la labor de todos.

\section{Agradecimientos}

Los autores quieren mostrar su agradecimiento al Vicerrectorado de Estudios, Calidad y Acreditación de la Universitat Politècnica de València por la financiación obtenida a través del programa PIME 2018-19 bajo la referencia DPTO.IMM.

\section{Referencias}

BARROWS, H.S. (1986). "A Taxonomy of problema-based learning methods” en Medical Education, 20 (6), p. 481486 .

CARBAllEIRA, J., ROVIRA, A., SUÑER, J.L., NADAL, E., RUPÉREZ, M.J., DOLS, J.F., SAHUQUILLO, O., MARTÍNEZ-CASAS, J., VILA, P., PEDROSA, A., DENIA, F.D., RÓDENAS, J.J., y TUR, M. (2017). "Diseño de actividades y uso de la coevaluación para fomentar el desarrollo de competencias transversales en ingeniería mecánica y de materiales". Instituto Ciencias de la Educación del Universitat Politècnica de València. En Congreso Nacional de Innovación Educativa y Docencia en Red (IN-RED 2017). Valencia, Spain: Editorial UPV.

CATEDRA STADLER (2020). Nuevas ideas para el diseño de estructuras de capotas y cabinas. https://www.catedrastadler.com/copia-de-convocatoria-de-trabajos [Consulta: 23 marzo 2020]

METTAS, AC., y CONSTANTINOU, CC. (2007). "The technology fair: a project-based learning approach for enhancing problem solving skills and interest in design and technology education" en International Journal of Technology and Design Education, 18, p. 79-100.

UNIVERSITAT POLITÈCNICA DE VALÈNCIA (2014). Proyecto institucional sobre Competencias Transversales. http://www.upv.es/contenidos/COMPTRAN/ [Consulta: 27 junio 2018] 
VILLA, A., y POBLETE, M. (2011). "Evaluación de competencias genéricas: principios, oportunidades y limitaciones" en Bordón. Revista de pedagogía, 63(1), p. 147-170.

YE-LIN, Y., PRATS-BOLUDA, G., GARCÍA-CASADO, J., GUIJARRO ESTELLÉS, E., y MARTÍNEZ-DEJUAN, J.L. (2017). "Análisis del empleo de la metodología aprendizaje basado en proyectos como herramienta de desarrollo y evaluación de múltiples competencias transversales. Aplicación en grupos numerosos de asignaturas en la rama de ingeniería". Instituto Ciencias de la Educación del Universitat Politècnica de València. En Congreso Nacional de Innovación Educativa y Docencia en Red (IN-RED 2017). Valencia, Spain: Editorial UPV. 